\title{
The relevance of local magnetic records when using extreme space weather events as benchmarks
}

\author{
Elena Saiz*, Consuelo Cid, and Antonio Guerrero \\ Universidad de Alcalá, Dpto. Física y Matemáticas, Space Weather Research Group, 28801 Alcalá de Henares, Madrid, Spain
}

Received 17 December 2020 / Accepted 12 May 2021

\begin{abstract}
Space weather indices introduced for scientific purposes are commonly used to quantify operational nowcast of the geospace state during extreme space weather events. Some indices, such as the Disturbance storm time (Dst) index, have been applied to situations for which they are not originally intended. This raises a question about suitability as a space weather benchmark. In analysing historical records for different magnetometers at low- and mid-latitude, we find periods with longitudinal asymmetry in magnetic response that suggest important signals from individual magnetometers are being averaged out of the Dst record. This asymmetry develops as a double spike in the H-component: one negative in the observatories in the day sector and one positive in the observatories in the night sector. These spikes develop in short-time (about $2 \mathrm{~h}$ ) and pose a potential hazardous effect for users affected by space weather. The results from historical events have been reinforced with the systematic study of magnetic records during extreme events $(D s t \leq-200 \mathrm{nT}$ and $A L \leq-2000 \mathrm{nT})$ in the period 1998-2017 from six magnetic observatories at about $40^{\circ}$ magnetic latitude. Moreover, we show that the largest asymmetries take place during the early main phase and are recorded in narrow local time sectors. An important outcome of these results is that space weather benchmarks should be based on local records instead of the commonly used global indices. This action improves two important aspects of space weather: the assessment of historical extreme events and that of the needs of users.
\end{abstract}

Keywords: Local magnetic records / H-spikes / Extreme events / Longitudinal asymmetry

\section{Introduction}

The relevance of space weather as a natural hazard is undeniable as it can damage critical infrastructure and technology systems. To enhance resilience, different benchmarks are commonly identified, which are expected to describe the nature and intensity of extreme space weather events, providing a point of reference from which to improve understanding of the effects of space weather (see some examples at https://www.sworm.gov/ publications/2018/Space-Weather-Phase-1-Benchmarks-Report. pdf).

Reconstructing the timeline of historical extreme events with spectacular technological impacts (Knipp et al., 2018; Hapgood, 2019; Hayakawa et al., 2020) and/or with extremely bright aurora witnessed at low magnetic latitudes (Ebihara et al., 2017; Kataoka et al., 2017) similar to the Carrington event in

\footnotetext{
*Corresponding author: elena. saiz@uah. es
}

September 1859 (Cliver \& Svalgaard, 2004; Cliver \& Dietrich, 2013) is considered as an excellent basis for space weather scenarios. These events are commonly associated with solar ejections reaching the Earth after a short transit time where the lesser value of the geomagnetic Dst index the larger the impacts. For example, Hayakawa et al. (2020), noted that during the space weather event in 1903, the telegraph communication network interruption in the Iberian Peninsula occurred during the maximum disturbance, where the Dst intensity estimated by the authors surpassed $-400 \mathrm{nT}$.

Among these extreme events, driving ultra-fast (often consecutive) solar ejecta, the 4-5 August 1972 storm has been seen as part of a grand challenge to understand this class of events by several authors (Kawasaki et al., 1973; Lakhina et al., 2013; Knipp et al., 2018) and therefore should be considered as a benchmark. Indeed, the August 1972 event, which had a significant civilian and military impact, was classified by Kawasaki et al. (1973) "among the most intense ever recorded" but the Dst index dipped only to $-125 \mathrm{nT}$ leaving this event out of the extreme geomagnetic storms lists and, as a consequence, 
probably out as a benchmark. Kawasaki et al. (1973) noted the large growth of the longitudinally asymmetric disturbance (ASY) index following the storm sudden commencement (SSC) at 20:54 UT on 4 August, caused by an abrupt decrease of the $H$-component at stations in the late morning sector. They indicated that similar unusual feature had been reported by Fukushima \& Abe (1958) and Akasofu \& Chapman (1960) during great magnetic storms, although they also suspected that the complexity of the low-latitude records might have been introduced by timing errors. Manila Observatory records (Salcedo, 1973; Knipp et al., 2018) provide an example of this abrupt decrease on 4 August 1972 of the $H$-component at a lowlatitude observatory located at that time at about six Magnetic Local Time (MLT), but what about the records from magnetometers at other MLT sectors different from the morning one? Does an abrupt decrease of the $H$-component also appear? Do unusual features appear during other great magnetic storms at low- or mid-latitude?

Cid et al. (2015) showed that the abrupt decrease of the $H$-component on 29 October 2003 reaching about $700 \mathrm{nT}$ at one mid-latitude observatory in the late morning sector (which they called $H$-spike), strongly depends on MLT. Moreover, Saiz et al. (2016) raised awareness about the possibility of missing hazardous space weather disturbances when using the Dst index. The reason was supported by the fact that the large $H$-spike recorded at Colaba during the Carrington event, might disappear in the average of the $H$-component from observatories located at a wide longitudinal range of MLTs. Note, for example, that the first failure reported in the Swedish High-Voltage Power Transmission System during the 29-31 October 2003 storm happened during an $H$-spike, as seen by the $I L$ index (local variant of the $A L$ index), even if $D s t$ had not reached even -50 nT (Pulkkinen et al., 2005). More recently, Love et al. (2019a,b) noticed that low latitude geomagnetic disturbance during the superstorm of September 1909 and May 1921 exhibited extreme longitudinal (local time) asymmetry at the initial phase of the storm. Are those unusual features extendable to mid-latitude geomagnetic records?

From a comprehensive statistical study, Sugiura \& Chapman (1960) studied the morphology of geomagnetic storms classified by intensity. They determined what they called "the disturbance longitudinal inequality" and made an analysis in terms of spherical harmonics. Their results show that the asymmetric character of the $H$-component reaches its maximum value during the first few hours of storm time. Fukushima \& Kamide (1973) also noted that the greatest longitudinal asymmetry in the $H$-component appears in the early main phase of magnetic storms. Does the longitudinal asymmetry reach always its maximum value in the early main phase?

In this paper we are interested in answering the questions raised above. For this purpose our study is focused on the extreme longitudinal asymmetry, using the terminology by Love et al. (2019b), or on the spike-like magnetic disturbances, using the terminology by Saiz et al. (2016) searching, observed in low- to mid-latitude records of the $H$-component during extreme events. Analysis begins with scrutinizing historical extreme events (Sect. 2) and then extends, applying a methodological processing, to more recent extreme events observed at about $40^{\circ} \mathrm{N}$ magnetic latitude (MLAT) in Section 3. Section 4 describes and discusses our results. Finally, Section 5 includes our conclusions and a rationale for considering local records in space weather benchmarks.

\section{Looking over extreme historical events}

\subsection{The 4-5 August 1972 event}

Top panel in Figure 1 compares the $X$-component of the ground magnetic field recorded at Manila (Philippines) (MAN, $14.6^{\circ} \mathrm{N}, 121.2^{\circ} \mathrm{E}$ geographic coordinates) and the $H$-component recorded at San Fernando (Spain) (SFS, 36.7 ${ }^{\circ} \mathrm{N}, 354.1^{\circ} \mathrm{E}$ geographic coordinates) magnetic observatories for the August 1972 event. During the time shown in the figure, three SSCs at 1:19 UT, 2:20 UT and 20:54 UT on 4 August (marked by vertical dashed lines) have been identified by Ebro Observatory, which houses the International Service on Rapid Magnetic Variations created by the International Association of Geomagnetism and Aeronomy (IAGA). The time scale of SFS digitized data has been shifted 15 min to match the clear response to the SSCs at this station.

Soon after the third SSC (vertical line SC3), a large asymmetry appears considering both locations. Indeed, while MAN magnetogram presents a large decrease in the $X$-component reaching about $250 \mathrm{nT}$, SFS magnetogram presents at the same time an increase in the $H$-component reaching about $250 \mathrm{nT}$. This large $X$ - and $H$-components asymmetry of about $500 \mathrm{nT}$ agrees with the large growth of the $A S Y-H$ index (longitudinally asymmetry for the $H$-component) following the SSC at 20:54 UT on 4 August noted by Kawasaki et al. (1973). Records from Hermanus (HER, $-34.4^{\circ} \mathrm{S}, 19.2^{\circ}$ E geographic coordinates) and Kakioka (KAK, $36.2^{\circ} \mathrm{N}, 140.2^{\circ} \mathrm{E}$ geographic coordinates) magnetic observatories (bottom panel in Fig. 1) show the same kind of asymmetry of the $H$-component following the SSC at 20:54 UT caused by an abrupt decrease at KAK station, in the late morning sector, along with an abrupt increase at HER station, in the dusk-night sector.

The visual comparison of the profiles of both observatories in each panel and between both panels in Figure 1, reveals a good concordance regarding an abrupt but asymmetric enhanced magnetic disturbance during about $2 \mathrm{~h}$ following SC3, which weakens the timing errors hypothesis proposed by Kawasaki et al. (1973).

On the other hand, HER and KAK are two of the four observatories involved in the Dst index (in black in the figure). Nevertheless, the abrupt changes in the $H$-component observed in HER and KAK at that time interval disappear in the Dst index due to the averaging process in the longitude to compute the index. Thus, during the event on 4-5 August 1972, the $D s t$ index is missing a potentially hazardous situation clearly recorded by local magnetometers, showing large and fast variation of ground magnetic field. Large values of $\mathrm{d} B / \mathrm{d} t$ are associated with the appearance of geomagnetically induced currents (Pulkkinen et al., 2005; Ngwira \& Pulkkinen, 2019; Hapgood et al., 2021), and may be the cause of the widespread electricand communication- grid disturbances which plagued North America late on 4 August and to the nearly instantaneous, unintended detonation of dozens of sea mines in North Vietman 


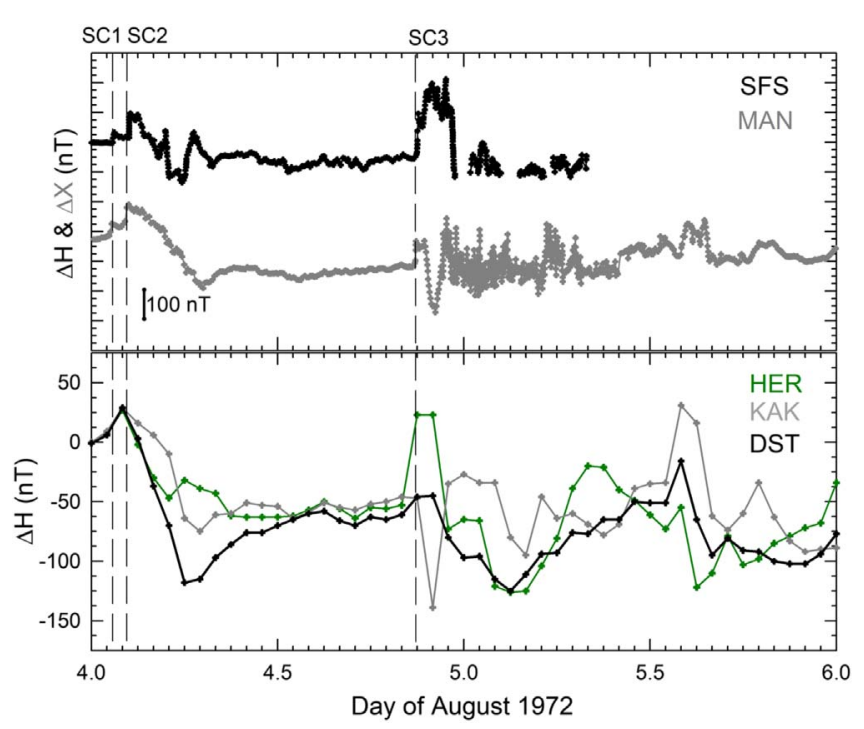

Fig. 1. Top panel shows the $X$ - and $H$-components recorded at Manila and San Fernando magnetic observatories, respectively, from 4 to 6 August 1972. Data at San Fernando have been digitized from the original source and data at Manila have been digitized from Salcedo (1973) giant. Scanned magnetograms are included in Appendix A. Bottom panel shows hourly data of the $H$-component recorded at Kakioka (KAK, grey) and Hermanus (HER, green) magnetic observatories and the Dst index (black) from Kyoto World Data Center.

(Knipp et al., 2018). The question to solve at this stage is the following: how unique is the event on 4 August 1972 regarding the longitudinal asymmetry of the $H$-component records at a low-mid latitude which is missed by the Dst index?

\subsection{The 13-14 July 1982 event}

A quick look of the magnetic disturbance of the $H$-component recorded at HER and KAK observatories during the magnetic storm on 13-14 July 1982 (Fig. 2) provides another clear example of the non-uniqueness of the event in August 1972. At 23:00 UT on 13 July an abrupt decrease of the $H$-component in KAK (in the late morning sector) dipped until $-525 \mathrm{nT}$, while the Dst index was about $-275 \mathrm{nT}$. In the meanwhile, records from HER (in the night sector at that time) showed an abrupt increase up to $+100 \mathrm{nT}$. Thus, at the time of maximum asymmetry (about $600 \mathrm{nT}$ ), the deviation of the $H$-component at KAK (HER) from the value of the Dst index at that time is $375 \mathrm{nT}$ $(-250 \mathrm{nT})$, a value larger than $130 \%(90 \%)$ of the Dst value.

\subsection{Other historical events with local spikes}

The signatures described above make the July 1982 event extremely similar to that of August 1972. The similarity between these two events, both regarding the large longitudinal asymmetry of the $\mathrm{H}$-component and regarding the local time of the observatory where the abrupt decrease and increase of the $H$-component appear (which we will name in what follows as negative-spike and positive-spike respectively), guided us to follow on the search for similar signatures in other historical events. Several historical records have been checked searching

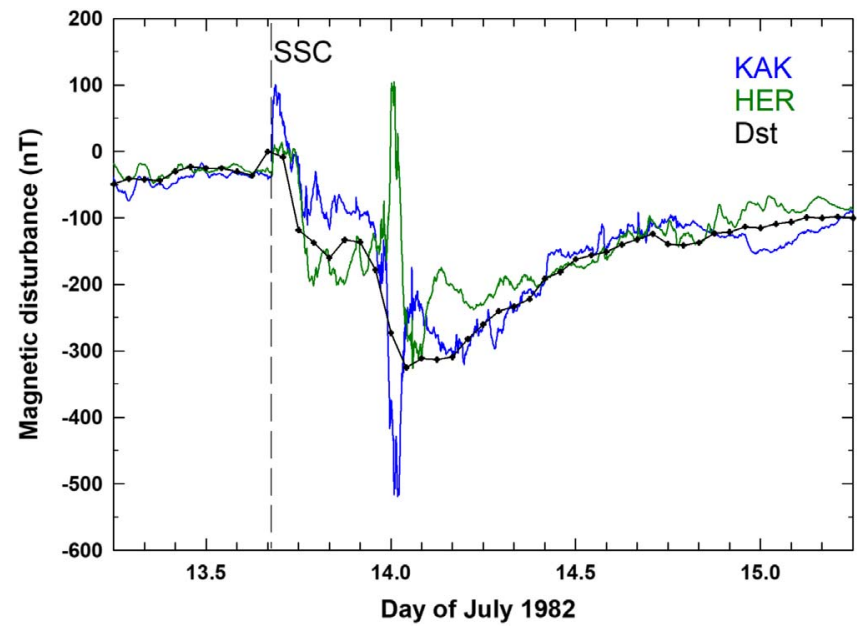

Fig. 2. $H$-component recorded at Kakioka (KAK, blue) and Hermanus (HER, green) geomagnetic observatories during the magnetic storm on 13-14 July of 1982. The Dst index (black) is also included for comparison with local records. The vertical dashed line corresponds to the time of the SSC of the storm.

for a double-spike configuration over the background magnetic field at the expected local time sectors, or at least one spike when not enough data are available. For this study, the sources of historical records have been the following:

- Digital images of the original magnetogram during historical geomagnetic storms recorded in different Spanish observatories provided by the Instituto Geográfico Nacional (https:// www.ign.es/web/ign/portal/gmt-tormentas).

- Digital images of the original magnetogram during historical geomagnetic storms recorded in different UK observatories and Yearbooks provided by the British Geological Survey (https://www.bgs.ac.uk/information-hub/scanned-records/ magnetograms/).

- Plots, data and original magnetograms (depending on the date) from Kyoto World Data Center (http://wdc.kugi.kyotou.ac.jp/).

- Figures in several references (see the last column of Table 1) dedicated to the analysis of historical events.

- Supermag tool for joint visualization of the magnetic field records from all the observatories available. This tool is useful from 1970 onward (http://supermag.jhuapl.edu/ $\mathrm{mag} /$ ).

Table 1 summarizes the historical events analyzed. The results in columns 8 and 10, which correspond to the MLT hourly resolution - of the center of the interval in column 5-15 min resolution-, reveal that all the negative (positive) spikes are recorded in observatories located in the day (night) sector (geographic and magnetic location of magnetic observatories in the table appear in Appendix B). Note that the lack of data from observatories distributed in longitude for this analysis does not allow us to determine the MLT where the negativespike and positive-spike have the maximum intensity. There is also a common signature in all the events: the duration of the double-spike event (see column 6) is about $1-2.5 \mathrm{~h}$, with an average value of $1.5 \mathrm{~h}$. 
Table 1. List of historic events (location information is given in Appendix B).

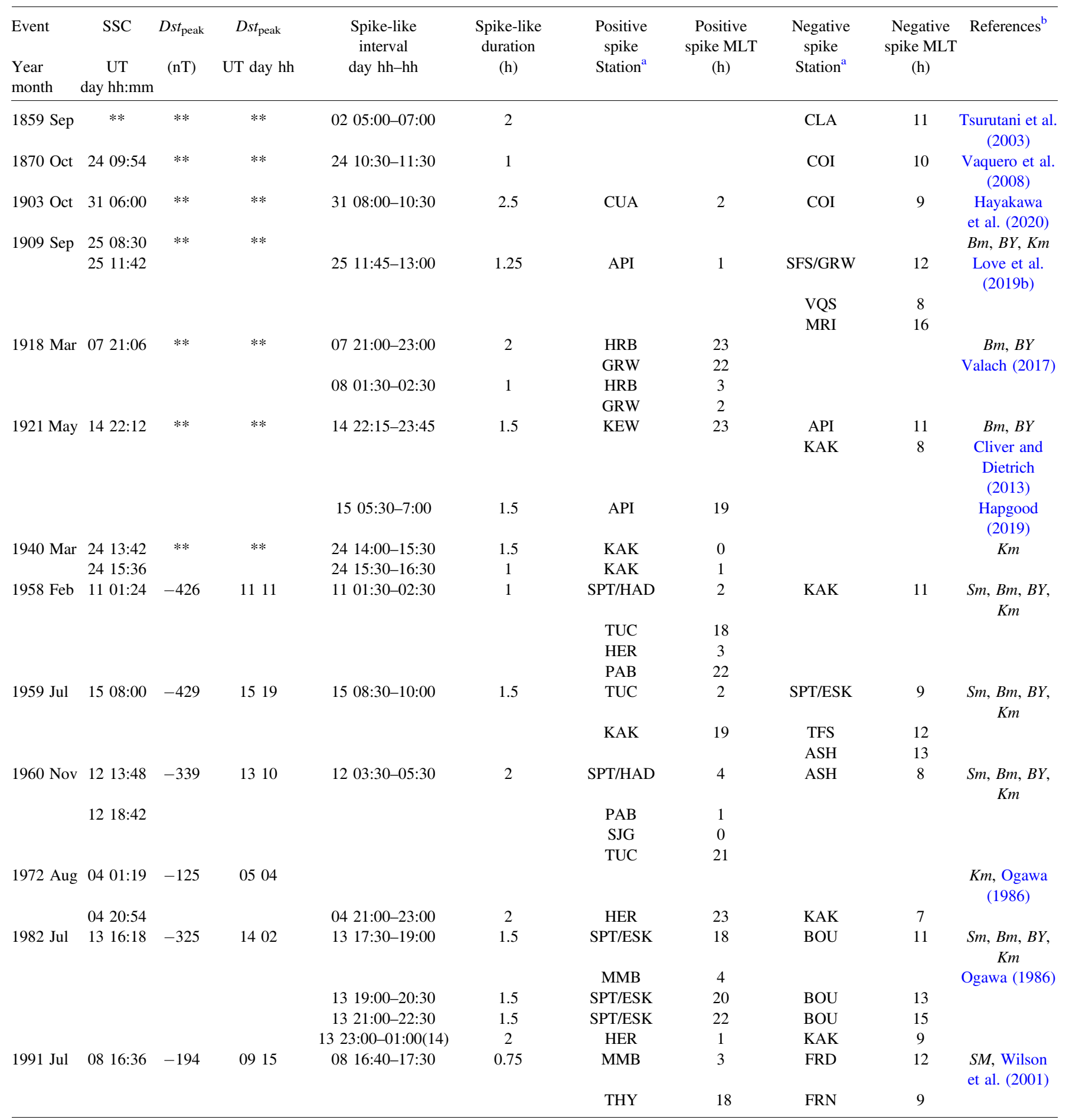

\footnotetext{
a IAGA code.
}

b Sm: Spanish magnetograms, Bm: British magnetograms, BY: British Yearbooks, Km: Kyoto magnetograms, SM: SuperMag.

Comparing the timing of the SSC (column 2, from http:// www.obsebre.es/en/rapid) and the time of the double-spike (column 5) for the events of Table 1, we notice that most of the events (10 out of 12, i.e. $83 \%$ ) develop the double-spike configuration (i.e. temporal overlapping positive and negative spike at different locations) very close to the SSC, in the very early main phase of the storm. This result agrees with Sugiura \& Chapman (1960) and Fukushima \& Kamide (1973). On the other hand, the double-spike event in Figure 2 takes place at the late main phase. 
Table 2. Magnetic observatories used in the study. The fifth column refers to the interval in which the magnetic latitude is included according to 2000-2015 IGRF models during the period analysed.

\begin{tabular}{lccccc}
\hline Observatory name & IAGA code & Geographic latitude $\left(^{\circ}\right)$ & Geographic longitude $\left(^{\circ}\right)$ & Magnetic latitude $\left(^{\circ}\right)$ & UT-MLT $(\mathrm{h})$ \\
\hline San Pablo-Toledo & SPT & 39.55 & 355.65 & $42.5 \pm 0.5$ \\
Surlari & SUA & 44.68 & 26.55 & $42.0 \pm 0.5$ & -0.29 \\
Irkutsk & IRT & 52.27 & 104.45 & $42.0 \pm 0.5$ & +1.77 \\
Memambetsu & MMB & 43.91 & 144.19 & $35.5 \pm 0.5$ & +6.96 \\
Fresno & FRN & 37.09 & 240.28 & $43.0 \pm 0.5$ \\
Stennis Space Center & BSL & 30.35 & 270.36 & $39.5 \pm 0.5$ \\
\hline
\end{tabular}

\section{Systematic analysis of recent data}

\subsection{Selection of magnetic observatories}

Considering the potentially hazardous effects of $H$-spikes, the results obtained from the previous section guided the authors to perform a systematic study of the longitudinal asymmetries in the $H$-component recorded at mid-latitude observatories at different MLTs. This study at mid-latitude location was designed to give a response to the needs of space weather users located on the Spanish mainland, about $40^{\circ}$ N MLAT, where the authors of this paper are playing major roles at the Spanish Space Weather Service (SeNMEs, http://www.senmes.es/indexen.php). To achieve this goal, we analyze extreme storms with data from a wide range of MLTs and with enough temporal resolution to be able to distinguish the signatures of spikes that last about $1.5 \mathrm{~h}$. This condition forces consideration of magnetic observatories with a good temporal data coverage and widely distributed in longitude (covering all sectors). Unfortunately, there are extensive areas (i.e., Pacific and Atlantic oceans) where no observatory is recording magnetic field data. To minimize any possible bias in data, we have selected six geomagnetic observatories from INTERMAGNET network (Table 2, Fig. 3). This selection of observatories ensures at least one observatory being located on each 6-h MLT sector (0-6, 6-12, 12-18 and 18-24 MLT). They are all located on a narrow latitude range: $35^{\circ}-45^{\circ} \mathrm{N}$ MLAT, providing the best coverage in MLT while keeping a roughly constant magnetic latitude.

\subsection{The sample of storms}

Dst and Auroral electrojet $(A E)$ (derived from the upper $(A U)$ and lower $(A L)$ envelopes) indices are commonly used by the scientific community to quantify the geomagnetic disturbance at low and high latitude, respectively. Indeed, they are proxies of the enhancement of the main currents at those latitudes: the ring current and the auroral electrojet, respectively. During extreme events the disturbance due to both currents may reach mid-latitude. Consequently, we will consider those events which are extreme at both low-latitude and high-latitude. Thus, we combine Dst and $A L$ data from Kyoto World Data Center (WDC) from 1998 to 2017 searching for candidates to extreme events. We consider an extreme event when Dst $\leq-200 \mathrm{nT}$ and $A L \leq-2000 \mathrm{nT}$. These two conditions have to be fulfilled along the interval of the storm event, but thresholds for Dst and $A L$ do not need to be passed simultaneously. After this systematic search, a preliminary list of events is obtained.

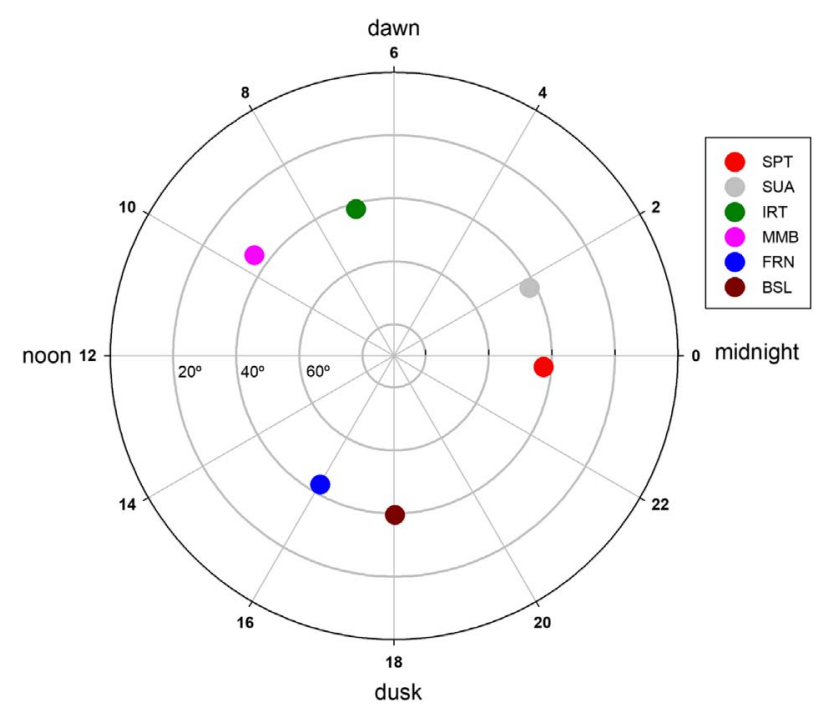

Fig. 3. Distribution of the six magnetic observatories selected. The MLT location shown corresponds to 00:00 UT.

The preliminary list needs to be filtered out to remove those events without continuous data available in all six observatories with one-minute resolution. An event is discarded if there is a data gap longer than $1.5 \mathrm{~h}$ (similar to the spike duration of the events in Table 1). The final list of events analysed in this paper appears in Table 3. The start and end days (4th and 5th columns in Table 3) have been fixed to cover the whole event.

\subsection{Data processing}

To properly analyse any asymmetry in the disturbance of the $H$-component it is important to accurately remove the solar quiet variation from observatory data before proceeding with an analysis of the disturbance (Love \& Gannon, 2010). We compute the solar regular variation pattern at every station, $S q_{\mathrm{obs}, i}$, as the average of the H-component of the magnetic field measured at that observatory during the five International Quiet Days (IQDs) of the month of the event (http://wdc.kugi.kyotou.ac.jp/qddays/index.html). We also evaluate the linear change between the first and last value for the local day and subtract it from the data, allowing us to join more than one day of data together.

Once we know $S q_{\mathrm{obs}, i}$, we compute the magnetic disturbance in the $H$-component at the observatory "obs" during the event $i, i=1$ to $10\left(H_{\mathrm{obs}, i}\right)$ with the following equation: 
Table 3. List of extreme events at mid-latitude according to the following criteria: $D s t \leq-200 \mathrm{nT}$ and $A L \leq-2000 \mathrm{nT}$. Availability of local magnetic field data from all six observatories in Table 1 is also mandatory.

\begin{tabular}{|c|c|c|c|c|c|c|}
\hline Event \# & Year & Month & Start day & End day & $D s t_{\text {peak }}(\mathrm{nT})$ & $A L_{\text {peak }}(\mathrm{nT})$ \\
\hline 2 & 2000 & July & 15 & 16 & -301 & -2900 \\
\hline 3 & 2000 & August & 10 & 13 & -235 & -2500 \\
\hline 5 & 2001 & November & 5 & 7 & -292 & -2800 \\
\hline 6 & 2001 & November & 24 & 25 & -221 & -3100 \\
\hline 7 & 2003 & October & 29 & 31 & -353 & -3900 \\
\hline 10 & 2015 & March & 17 & 18 & -222 & -2300 \\
\hline
\end{tabular}

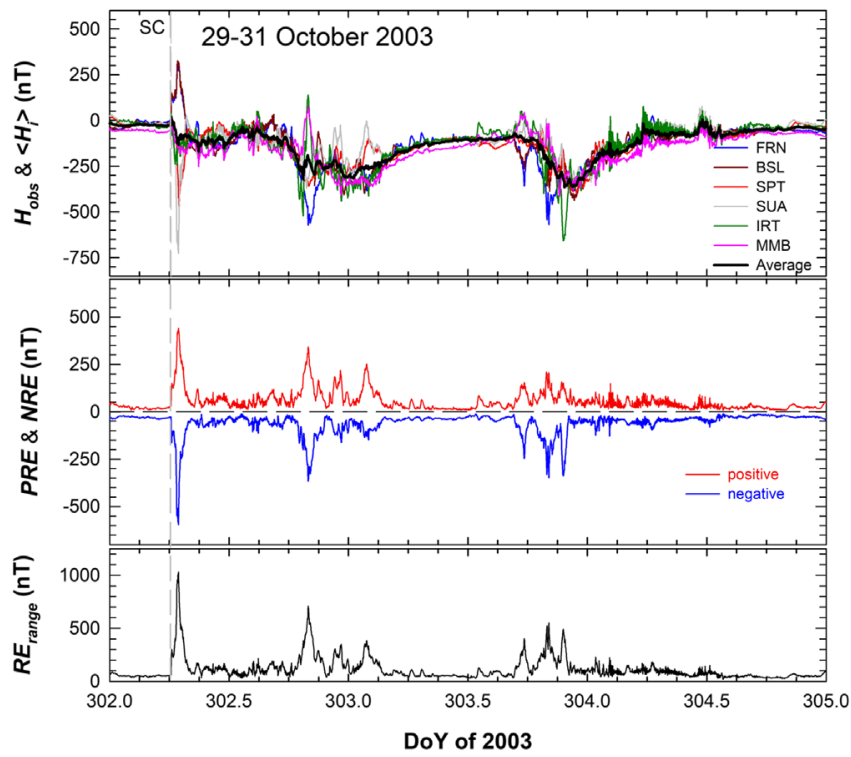

Fig. 4. The event on 29-31 October 2003 as an example of processing. Top panel: Magnetic disturbance, $H_{\mathrm{obs}}$, at the midlatitude observatories in Table 1 (in colours) and the average of the disturbance $\left\langle H_{i}\right\rangle$ (in black); Second panel: Positive (red) and Negative (blue) Residual Envelopes; Bottom panel: Residual Envelopes range as a proxy for the asymmetry intensity. Grey dashed line marks the SSC in the period plotted.

$$
H_{\mathrm{obs}, i}(t)=H(t)-\mathrm{Sq}_{\mathrm{obs}, i}
$$

with $H(t)$ representing the $H$-component of the magnetic field measured at observatory "obs" (with obs $=2$ nd column of Table 2).

Then we compute for every event $i$ the average of the disturbance, $\left\langle H_{i}\right\rangle$, and the residual of the disturbance at every observatory relative to the average, $R_{\mathrm{obs}, i}$ :

$$
\begin{gathered}
\left\langle H_{i}(t)\right\rangle=\frac{\sum_{\mathrm{obs}} H_{\mathrm{obs}, i}(t)}{6} \\
R_{\mathrm{obs}, i}(t)=H_{\mathrm{obs}, i}(t)-\left\langle H_{i}(t)\right\rangle .
\end{gathered}
$$

$\left\langle H_{i}\right\rangle$ can be seen as a proxy for the symmetrical magnetic disturbance of the $H$-component at about $40^{\circ} \mathrm{N}$ MLAT (similar to

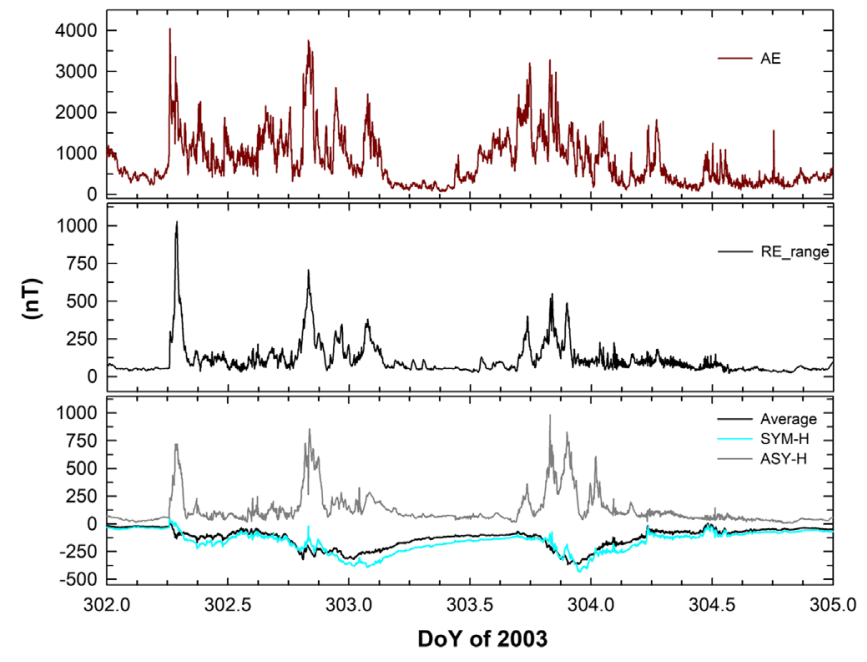

Fig. 5. Comparison between different indices for the event on 29-31 October 2003. Top panel: $A E$ index; Second panel $R E_{\text {range }}$; Bottom panel: $A S Y-H$ (grey), $S Y M-H$ (cyan) and the average of the disturbance $\left\langle H_{i}\right\rangle$ (black).

$S Y M-H$ index), and $R_{\mathrm{obs}, i}$ is a measurement associated with the local asymmetric disturbance. Top panel in Figure 4 shows, as an example, the $H$-disturbance at every observatory (in colours), as defined in equation (1), and the average of the $H$-disturbance, $\left\langle H_{i}\right\rangle$ (in black) during the event on 29-31 October $2003(i=3$, event \#7). Bottom panel of Figure 5 compares $\left\langle H_{i}\right\rangle$ (in black) with the $S Y M-H$ index (in cyan) showing the similarity between both indices. Figures for the other extreme events are included in Appendix C.

The next step is to compute the envelopes of the residuals $R_{\text {obs }, i}$. We calculate the Positive Residual Envelope (PRE) and Negative Residual Envelope (NRE) as the maximum and minimum value of the residuals at each minute, respectively, from the six observatories analysed. Both envelopes can be considered to be analogues to the high-latitude $A L$ and $A U$ indices, but for about $40^{\circ} \mathrm{N}$ MLAT, with the advantage of having removed the problem of the regular variation that $A L$ and $A U$ indices have (Mayaud, 1980, Sect. 7.4, p. 106). Second panel in Figure 4 shows the envelopes of the residuals for the event in October 2003.

Finally, the residual envelopes range, $R E_{\text {range }}$ (bottom panel in Fig. 4), measures the range between the Positive and Negative 
Table 4. Summary of the results for the events analysed. The table includes: the peak value reached by the $R E_{\text {range }}($ column 2 ) for the event (column 1), the date (UT) when the peak value of $R E_{\text {range }}$ is reached (column 3), the Positive and Negative Residual Envelopes (PRE and NRE) at that date (columns 4 and 6) and the MLT at the observatory whose $R_{\mathrm{obs}, i}$ coincides with the PRE and NRE at the date in column (3).

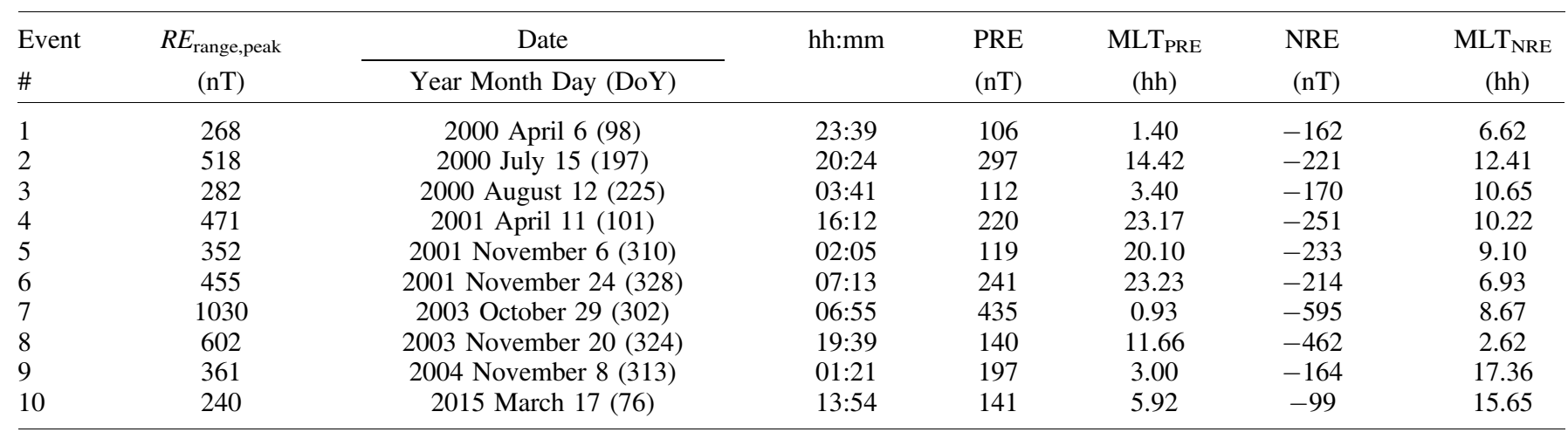

Residual Envelope $\left(R E_{\text {range }}=\mathrm{PRE}-\mathrm{NRE}\right)$ and therefore it is conceptually similar to $A E$ or $A S Y-H$ index but for about $40^{\circ} \mathrm{N}$ MLAT. $R E_{\text {range }}$ will be zero if the disturbance would not depend on the longitude. Thus, $R E_{\text {range }}$ can be considered as a proxy for the magnitude of the longitudinal asymmetry.

At this point we highlight that we are comparing $R E_{\text {range }}$ to $A E$ or $A S Y$ - $H$ indices only as proxies to quantify the longitudinal asymmetry at different latitudes, but at this stage we are not searching for the physical explanation for the $R E_{\text {range }}$ We are aware that $A S Y-H$ is considered as the metric for the asymmetric ring current, while $A E$ is the metric for the strength of the substorm current wedge, providing in this way a physical explanation for the asymmetric disturbance at different latitudes based on different magnetospheric currents. However, the association of $R E_{\text {range }}$ with a metric for any magnetospheric current is out of the scope of this paper. Nevertheless, Figure 5 allows a comparison between $R E_{\text {range, }} A E$ and $A S Y$ - $H$ indices. As can be appreciated in the Figure, the $A S Y-H$ index holds most of the

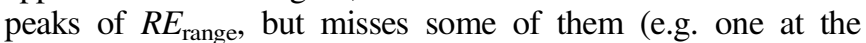

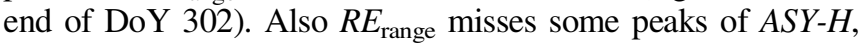
as that at the very beginning of DoY 304. However no apparent relationship appears between the intensity of the peaks at low and mid-latitude. This fact is evidenced, for example, when comparing the peaks at DoY 302 starting at about 06 UT and about 20 UT. Both have similar intensity in $A S Y-H$, while the peaks in $R E_{\text {range }}$ are clearly different. The $A E$ index also captures most of the peaks in $R E_{\text {range, including some missing }}$ by $A S Y-H$ as those close to midnight straddling the DoY 302 and 303 , suggesting that $R E_{\text {range }}$ may be measuring a mix of the strength of currents at low and high latitude. The currents involved in $R E_{\text {range }}$ appears as an interesting study which deserves our attention for a future study.

\section{Results and discussion}

Table 4 shows the maximum value of $R E_{\text {range }}$ (column 2) for each of the events in Table 4 and the date (UT) when it happens (column 3), i.e, the date of the maximum asymmetry observed for that event. Columns 4-7 in Table 4 display the values of PRE and NRE for that date and the MLT of the observatories whose $R_{\mathrm{obs}, i}$ coincides with PRE and NRE at the date in column (3). Figures for all events analysed (except the event \#7) are provided in Appendix C.

Making a comparison between the peak value of the $R E_{\text {range }}$ (Table 4, column 2) and that of the $D s t_{\text {peak }}$ (Table 3, column 6) for the events analysed, we obtain that the peak value of the $R E_{\text {range }}$ is larger than the $D s t_{\text {peak }}$ for all the events but two (events \#1 and \#9). For these two events, the difference is less than $10 \%$, indicating still a large asymmetry when comparing local records of the disturbance in the $H$-component. Moreover, in some cases (events \#6 and \#7), this difference is over $100 \%$ of the $D s t_{\text {peak }}$ value. In all cases, the peak value of the $R E_{\text {range }}$ corresponds to the minimum/maximum value of an abrupt decrease/increase of the $H$-component, as seen by the NRE and PRE (see Fig. 4 as an example). Even though not shown in Table 4 , the duration of the $H$-spikes resulting in the peak value of the $R E_{\text {range }}$ is always less than $3 \mathrm{~h}$. This duration is similar to the value obtained for the historical events in Section 2. These results indicate that these extreme events exhibit extreme longitudinal asymmetry and, as a first consequence, local records are crucial and cannot be disregarded when using the extreme events as benchmarks.

Examining the phase of the storm, we obtain that the maximum longitudinal asymmetry occurs during the early main phase only in four out of the ten events analysed in Table 3. Specifically, the peak value of the $R E_{\text {range }}$ takes place less than one hour and a half after the SSC in these four events. The result for these four events and for most of the events in Table 1 is in agreement to the results by Sugiura and Chapman (1960) and Fukushima and Kamide (1973) about the occurrence of the spike a few hours from the SSC. Nevertheless, there is more than $50 \%$ of events in Table 3 and a few others in Table 1 with the largest asymmetry appearing far from the SSC. The event in July 1982 (Fig. 2) is an example of this type of events.

Figure 6 allows us to check for any dependence on local time of the maximum asymmetry for the events analysed, which can be also deduced from the values in columns 5 and 7 in Table 4. Those events whose maximum asymmetry takes place during the early main phase (marked by stars in Fig. 6), happen in a narrow local time segment: negative spike (in blue) in 7-10 MLT and positive spike (in red) in 20-01 MLT. Also, these events have the most intense values of both PRE and 


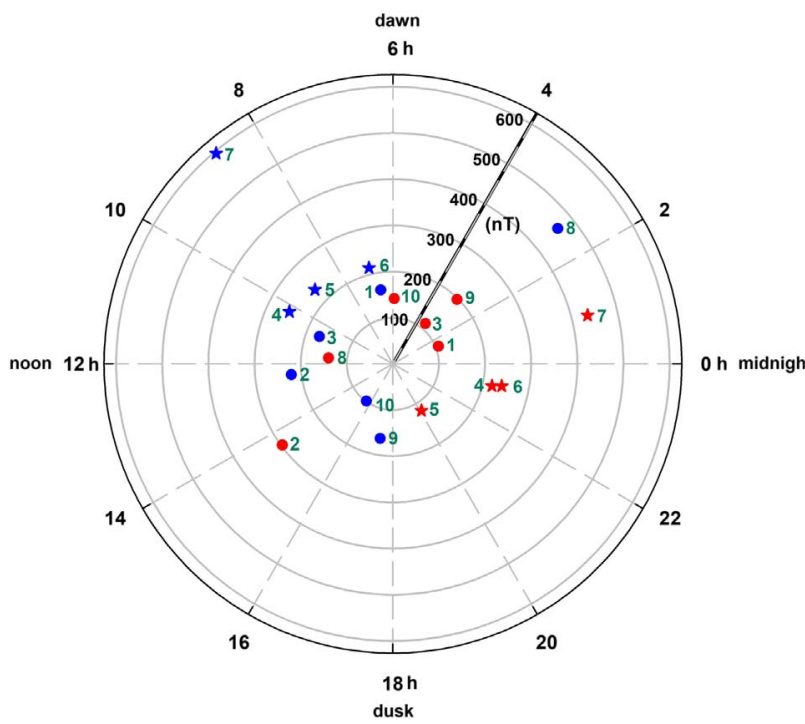

Fig. 6. MLT distribution of the Positive Residual (red) and Negative Residual (blue) for all events in Table 3 at the time of the maximum asymmetry as measured by $R E_{\text {range }}$. Note that NRE has been considered in absolute value. Stars mark those events whose maximum asymmetry takes place during the early main phase of the storm.

NRE (always larger than $200 \mathrm{nT}$ ). An extreme negative asymmetry of the $H$-component at about 09 MLT during the early main phase of the Bastille Day storm (event \#2 of the list) and that of the Halloween storm (event \#7) was already noticed by Love and Gannon (2010).

Considering the whole sample of events in Table 4, most of NRE values (blue symbols in Fig. 6) were recorded during the daytime sector (6-18 MLT), with a maximum concentration of about 7-11 MLT, while most of PRE values (red symbols) were recorded in the night time sector (18-6 MLT), with a maximum concentration about 23-4 MLT. This day-night pattern for negative-positive $H$-spikes was already noticed in the results from the analysis of the historical events in Section 2. Nevertheless, two events in Figure 6 appear as "outliers" of the pattern: the event on 20-21 November 2003 (event \#8), with a positive (negative) $H$-spike happening about 12 MLT (2.5 MLT), and the event on 15-16 July 2000 (event \#2), with a positive spike happening at 14.5 MLT -negative spike for this event is in the expected sector according to the day-night pattern.

The event on 15-16 July 2000 (see Fig. 7) presents its maximum asymmetry according to $R E_{\text {range }}$ at 20:24 UT on 15 July, during the late main phase. However, the large decrease (increase) appearing in NRE (PRE) cannot be labeled as an abrupt decrease (increase) but as a gradual one, contrary to those previously described in the so-called double spike events. Indeed the duration of the asymmetry peaking at 20:24 UT is larger than the typical duration of 1-2 h. Nevertheless, during this geomagnetic storm, there are other two significant longitudinal asymmetries that stand out in Figure 7, which are clearly double-spike events considering the sharpness of the NRE and PRE and their duration. These two spike-events happen at about 15 UT and 17 UT. During the spike-event at 15 UT, the observatories providing the records contributing to the PRE and NRE are, respectively, IRT and BSL. Therefore, in this case, the negative spike was recorded at about 9 MLT and the positive one at 22 MLT. On the other hand, during the event at 17 UT, the negative spike was recorded at FRN, at 9 MLT as the previous event, and the positive one at SUA (at about 19 MLT). Both double-spike events clearly follow the day-night pattern previously identified in Figure 6 and occur during the early main phase. Indeed an extreme negative asymmetry of the $H$-component was already identified by Love \& Gannon (2010) for this geomagnetic storm corresponding to that happening at about $15 \mathrm{UT}$ at BSL magnetic observatory.

The other event pointed out above as outlier is the event \#8 (20-21 November 2003). Two large negative $H$-spikes were recorded at IRT observatory while in the night sector, which are out of the ordinary when compared to the $H$-component of the other five mid-latitude observatories (see Fig. C.6). Indeed, the double-spike pattern is not appropriate to describe the features observed during any of the two peaks in $R E_{\text {range. }}$ Although the duration of the negative spikes is the expected (1-2 h), there is not a positive counterpart associated with any negative spike. Understanding this behaviour is out of the scope of this paper, but it deserves a dedicated analysis.

\section{Conclusions}

Several questions were raised in the Introduction section having as a base the records from Manila Observatory and its related consequences. After this study covering both historical and recent extreme events, we are ready to answer those questions.

The first questions, which were related to the abrupt decrease of the $H$-component at stations in the late morning sector previously reported by Kawasaki et al. (1973), Fukushima \& Kamide (1973), Akasofu \& Chapman (1960) and Knipp et al. (2018), were the following: what about the records from magnetometers at other MLT sectors different from the morning one? Does an abrupt decrease of the $H$-component also appear? Do unusual features appear during other great magnetic storms at low or mid-latitude?

Now, based on many examples, we can answer that magnetometers at other MLT sectors show also abrupt changes of the $\mathrm{H}$-component, but instead of an abrupt decrease, an abrupt increase usually appears at the night sector.

The next question was related to the extreme longitudinal asymmetry at the early main phase already noticed during some events (Cid et al., 2015; Saiz et al., 2016; Love et al., 2019a, 2019b). In those cases, the average of the $H$-component from observatories located at a wide longitudinal range of MLTs to obtain indices like Dst and $S Y M-H$ may miss the most important $H$-disturbances, as illustrated by Saiz et al. (2016) for the event on 21-22 January 2005. In this scenario, the question was: Are those unusual features extendable to mid-latitude geomagnetic records?

We have shown in this work numerous examples of extreme geomagnetic storms which present a common feature: a large longitudinal asymmetry of the $H$-component recorded at lowto-mid-latitude geomagnetic observatories, and therefore, those unusual features referred in this question are extendable to mid-latitude geomagnetic records. Moreover, the systematic analysis of recent data allows to conclude that the intensity of 


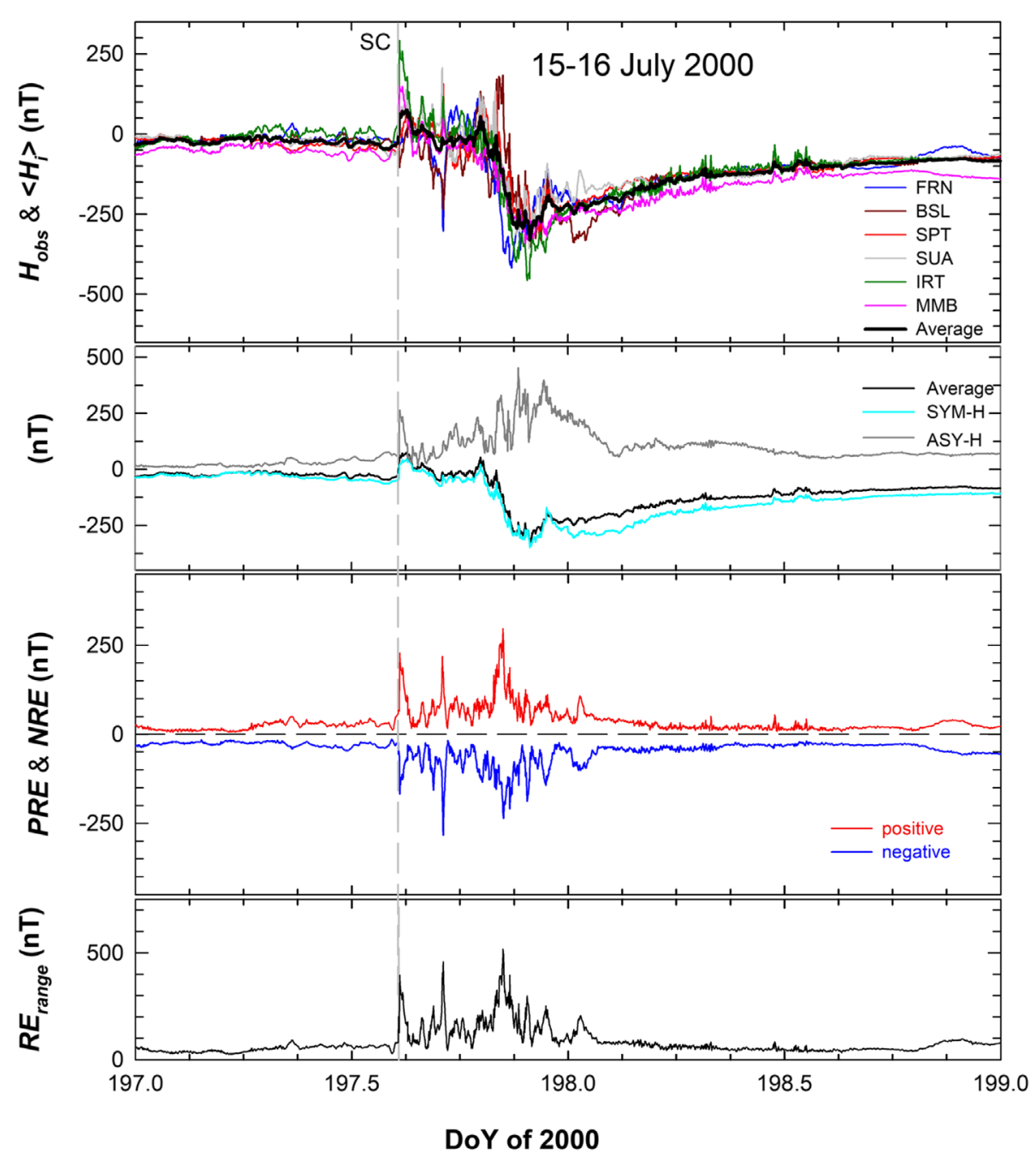

Fig. 7. The event on 15-16 July 2000 (event \#2). Top panel: Magnetic disturbance, $H_{\mathrm{obs}}$, at the six mid-latitude observatories (in colors) and the average of the disturbance, $\left\langle H_{i}\right\rangle$ (in black); Second panel: $\left\langle H_{i}\right\rangle$ (in black), $S Y M-H$ index (in cyan) and $A S Y-H$ index (in dark gray); Third panel: Positive (red) and Negative (blue) Residual Envelopes; Bottom panel: Residual range obtained as the difference between the Positive and Negative Residual Envelopes. Grey dashed line marks the SSC in the period plotted.

the asymmetry is at least comparable to the peak value of the storm, and sometimes its difference is more than $100 \%$. Furthermore, the longitudinal asymmetry develops as a double-spike (i.e., overlapping positive and negative $H$-spike on stations at different MLT), which recovers previous values in $1-2 \mathrm{~h}$ following a day-night pattern. These spikes disappear in the averaging process used to obtain commonly used geomagnetic indices, like the $S Y M-H$. This disappearance can be noticed when comparing local deviation in $H$-component at different observatories to the average in top panel in Figure 4 (or the figures in Appendix C, which include the $S Y M-H$ index, the average $H$-disturbance and the local $H$-disturbances). This type of asymmetry has been ignored or barely considered in space weather benchmarks. Nevertheless, such spikes are a major concern because of their potential to produce extreme geoelectric fields in some regions impacting contemporary technologies (e.g. see Fig. 2 in Saiz et al., 2016). A deeper analysis of the $H$-component records from magnetometers widespread in longitude could also enable a better assessment of the relevance of the minimum Dst value in historical storms, such as the Carrington event for which the index was estimated just from one magnetic observatory by ages. In this scenario, to consider local records when selecting events to be used as benchmarks appears as a must. Otherwise, space weather events with hazardous consequences may be missed as the $S Y M-H$ index (or the $D s t$ index) does not catch these disturbances.

Our previous answer was only related to the longitudinal asymmetry, but does it always happen at the early main phase? Sugiura \& Chapman (1960); Fukushima \& Kamide (1973) among some other authors noted that the greatest longitudinal asymmetry in the $H$-component appears in the early main phase of magnetic storms. In this scenario, our last question was the following: Does the longitudinal asymmetry always reach its maximum value in the early main phase?

The answer to this question is no, as the maximum asymmetry takes place during the early main phase only in about half of the events in the systematic analysis. Nevertheless, those events whose maximum asymmetry takes place during the early main 
phase are those with the most intense values of both PRE and NRE (always larger than $200 \mathrm{nT}$ ). Moreover, for some of the events with the maximum asymmetry out of the early phase, we can discover other large asymmetries in the early main phase. The spikes contributing to these asymmetries may be more relevant at higher latitudes if related to substorms. Records from magnetometers at higher latitudes were checked in Table 1 and also in the studies done by the quoted references. This fact may explain the partial disagreement between our results from Table 3 and those from Table 1 and the quoted references. In any case, the events in Table 3 with the maximum asymmetry taking place during the early main phase correspond to the most intense values of the residuals and therefore, are the most striking in a systematic study.

We would like to highlight that the timeline in space weather benchmarks is crucial. The availability of information about the time of the impacts on technological systems deserves a detailed analysis to be aware whether they are triggered by large local disturbances or by large values of typical indices. Further investigation is needed in this issue. Moreover, space weather scenarios using local geomagnetic disturbances may provide a valuable challenge, that of the MLT where the impact of the storm will be more hazardous.

Local records of $H$-component are showing a superposition of disturbances sustained by magnetopause currents, supported by usually large solar wind pressure during extreme events, partial ring currents and field-aligned currents. The contribution of each current system to produce the large longitudinal asymmetry of the $H$-component described in this paper and its solar wind trigger is another challenging issue nowadays.

To conclude, even if questions initially raised in the Introduction section have been answered throughout this paper and summarised in this section, this paper also shows that more research needs to be done regarding the observed longitudinal response in the $H$-component. Just as an example of the new questions to be solved we can mention the following: which are the effects behind the longitudinal asymmetry in local geomagnetic records? Are the more modern localized geomagnetic indices, such as the SuperMAG SMR-LT indices, able to properly catch those large longitudinal asymmetries? How are the longitudinal asymmetries affected, or how relevant are these asymmetries, during asymmetric driving of the magnetosphere? Will asymmetries in the ionosphere, or asymmetries in the local geology, help to explain some of the results? How relevant is the local geology relative to the solar wind drivers regarding the observed longitudinal asymmetry? We will try to answer these (and other) new questions in our follow-up research.

Acknowledgements. This work was supported by the MINECO project AYA2016-80881-P (including FEDER funds). We sincerely thank three anonymous reviewers for their helpful comments. We also acknowledge the following data sources: (1) the Dst and the $A L$ data obtained through the Kyoto WDC (http://wdc.kugi.kyoto-u.ac.jp/) and other plots and data from historical events, namely (2) the magnetic data from ground magnetometers in Table 2 freely accessible from the INTERMAGNET database (http://www.intermagnet.org/), (3) the original magnetogram provided by Dr. Manuel Catalan from the Real Observatorio Astronómico de la Armada in San Fernando (Spain) for the event in August 1972 (4) digital images of the original magnetogram during historical geomagnetic storms recorded in different Spanish observatories provided by the Instituto Geográfico Nacional (https:// www.ign.es/web/ign/portal), (5) digital images of the original magnetogram during historical geomagnetic storms recorded in different UK observatories and Yearbooks provided by the British Geological Survey (http://www.bgs.ac.uk/), and (6) the SuperMAG tool for joint visualization of the magnetic field records from different observatories (http://supermag. jhuapl.edu/). Finally, we thank the teams of the magnetic observatories who over the years have recorded the data used in this work. The editor thanks three anonymous reviewers for their assistance in evaluating this paper.

\section{References}

Akasofu S-I, Chapman S. 1960. Some features of the magnetic storms of July 1959 and tentative interpretations. In: Symposium of the July 1959 Events and Associated Phenomena, ISSN 05391016, IUGG Monograph, vol. 7. Institut geographique national (IGN), Paris. pp. 93-108.

Cid C, Saiz E, Guerrero A, Palacios J, Cerrato Y. 2015. A Carrington-like geomagnetic storm observed in the 21 st century. $J$ Space Weather Space Clim 5: A16. https://doi.org/10.1051/swsc/ 2015017.

Cliver EW, Dietrich WF. 2013. The 1859 space weather event revisited: limits of extreme activity. J Space Weather Space Clim 3: A31. https://doi.org/10.1051/swsc/2013053.

Cliver EW, Svalgaard L. 2004. The 1859 solar-terrestrial disturbance and the current limits of extreme space weather activity. Sol Phys 224(1-2): 407-422. https://doi.org/10.1007/s11207-0054980-z.

Ebihara Y, Hayakawa H, Iwahashi K, Tamazawa H, Kawamura AD, Isobe H. 2017. Possible Cause of Extremely Bright Aurora Witnessed in East Asia on 17 September 1770. Space Weather 15(10): 1373-1382. https://doi.org/10.1002/2017SW001693.

Fukushima N, Abe S. 1958. The Initial Phase of the Magnetic Storm on Feb. 11, 1958. In: Report of Ionosphere Research in Japan, vol. 12. Ionosphere Research Committee, Science Council of Japan. pp. 44-49. ISSN 0370-5773.

Fukushima N, Kamide Y. 1973. Partial ring current models for worldwide geomagnetic disturbances. Rev Geophys 11(4): 795853. https://doi.org/10.1029/RG011i004p00795.

Hapgood M. 2019. The great storm of May 1921: An exemplar of a dangerous space weather event. Space Weather 17(7): 950-975. https://doi.org/10.1029/2019SW002195.

Hapgood M, Angling MJ, Attrill G, Bisi M, Cannon PS, et al. 2021. Development of Space Weather Reasonable Worst Case Scenarios for the UK National Risk Assessment. Space Weather 19(4): 1-32. https://doi.org/10.1029/2020SW002593.

Hayakawa H, Ribeiro P, Vaquero JM, Gallego MC, Knipp DJ, et al. 2020. The Extreme Space Weather Event in 1903 October/ November: An Outburst from the Quiet Sun. Astrophys J 897(1): L10. https://doi.org/10.3847/2041-8213/ab6a18.

Kataoka R, Isobe H, Hayakawa H, Tamazawa H, Kawamura AD, et al. 2017. Historical space weather monitoring of prolonged aurora activities in Japan and in China. Space Weather 15(2): 392-402. https://doi.org/10.1002/2016SW001493.

Kawasaki K, Kamide Y, Yasuhara F, Akasofu S. 1973. UAG-28, part III, vol 3, World Data Center A, Boulder, CO. pp. 702-707. 
Knipp DJ, Fraser BJ, Shea MA, Smart DF. 2018. On the little-known consequences of the 4 August 1972 ultra-fast coronal mass ejecta: facts, commentary, and call to action. Space Weather 16(11): 1635-1643. https://doi.org/10.1029/2018SW002024.

Lakhina GS, Alex S, Tsurutani BT, Gonzalez WD. 2013. Supermagnetic Storms: Hazard to Society. American Geophysical Union (AGU), American Geophysical Union (AGU). pp. 267-278. https:// agupubs.onlinelibrary.wiley.com/doi/pdf/10.1029/2011GM001073.

Love JJ, Gannon JL. 2010. Movie-maps of low-latitude magnetic storm disturbance. Space Weather 8(6): 1-21. https://doi.org/ 10.1029/2009SW000518.

Love JJ, Hayakawa H, Cliver EW. 2019a. Intensity and impact of the New York Railroad superstorm of May 1921. Space Weather 17 (8): 1281-1292. https://doi.org/10.1029/2019SW002250.

Love JJ, Hayakawa H, Cliver EW. 2019b. On the intensity of the magnetic superstorm of September 1909. Space Weather 17(1): 37-45. https://doi.org/10.1029/2018SW002079.

Mayaud PN. 1980. Derivation, meaning, and use of geomagnetic indices, vol. 22, American Geophysical Union. ISBN: 0-87590022-4. https://doi.org/10.1029/GM022.

Ngwira CM, Pulkkinen AA. 2019. An Introduction to Geomagnetically Induced Currents, chap. 1, 1-13, American Geophysical Union (AGU). pp. 1-13. https://doi.org/10.1002/9781119434412.ch1.

Ogawa T. 1986. Solar-terrestrial disturbances of June - September 1982. III. Solar flares, flare particles and geomagnetic disturbances. Radio Res Lab J 33: 23-48.

Pulkkinen A, Lindahl S, Viljanen A, Pirjola R. 2005. Geomagnetic storm of 29-31 October 2003: Geomagnetically induced currents and their relation to problems in the Swedish high-voltage power transmission system. Space Weather 3(8): https://doi.org/ https://doi.org/10.1029/2004SW000123.

Saiz E, Guerrero A, Cid C, Palacios J, Cerrato Y. 2016. Searching for Carrington-like events and their signatures and triggers. J Space Weather Space Clim 6: A6. https://doi.org/10.1051/swsc/2016001.

Salcedo J. 1973. On the giant pulsations of the 04-06 August 1972 geomagnetic storm observed at Manila. in: UAG-28, Part III, World Data Center A, Boulder, CO. pp. 762-763.

Sugiura M, Chapman S. 1960. The Average Morphology of Geomagnetic Storms with Sudden Commencement. In: Contributions of the Geophysical institute. Series B (University of Alaska, College), No. 50. University of Alaska, Geophysical Institute.

Tsurutani BT, Gonzalez WD, Lakhina GS, Alex S. 2003. The extreme magnetic storm of 1-2 September 1859. J Geophys Res Space Phys 108: A7. https://doi.org/10.1029/2002JA009504.

Valach F. 2017. The intense magnetic storm of March 1918 as recorded by observatory Ógyalla (present day Hurbanovo). Acta Geod Geoph 52(4): 457-465. https://doi.org/10.1007/s40328-0160177-1.

Vaquero J, Valente M, Trigo R, Ribeiro P, Gallego M. 2008. The 1870 space weather event: Geomagnetic and auroral records. $J$ Geophys Res Space Phys 113: A8. https://doi.org/10.1029/ 2007JA012943.

Wilson GR, Burke WJ, Maynard NC, Huang CY, Singer HJ. 2001. Global electrodynamics observed during the initial and main phases of the July 1991 magnetic storm. J Geophys Res Space Phys 106(A11): 24517-24539. https://doi.org/10.1029/2000JA000348. 


\section{Appendix A}

\section{Historical magnetograms}

The magnetograms of Manila Observatory (Philippines) and San Fernando Observatory (Spain) appearing in the figures have been digitized and data are shown in the top panel of the Figure 1 of the manuscript.

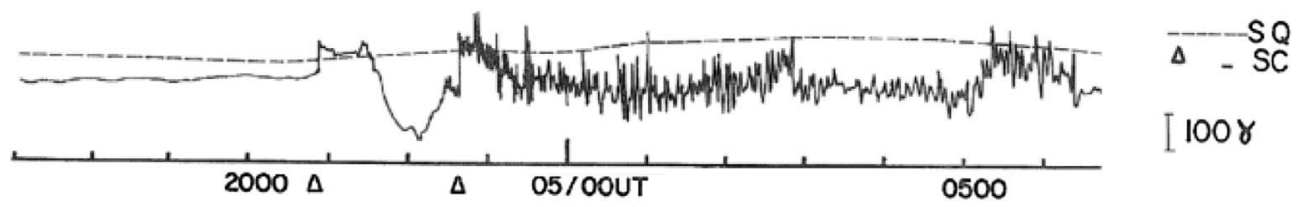

Fig. A.1. Manila Observatory magnetic $X$-component variations from August 4, 1972 at 17 UT to August 5 at 7 UT (Salcedo, 1973x).
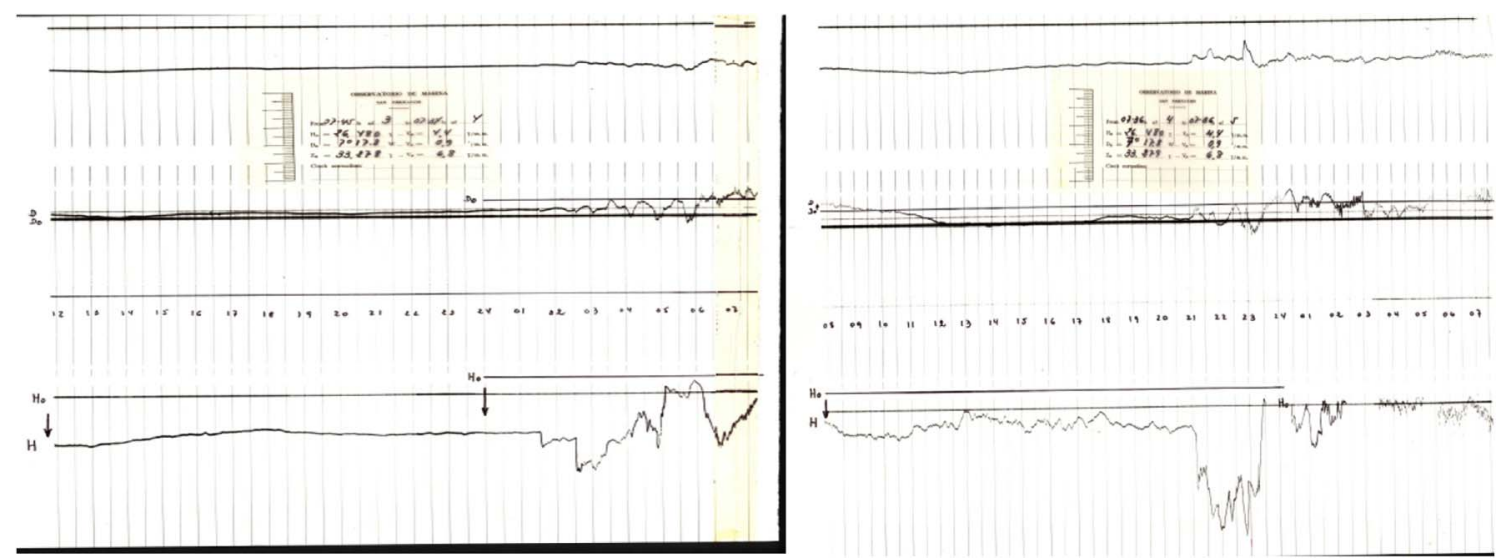

Fig. A.2. San Fernando Observatory $H$-component variations August 4, 1972 at 08 UT to August 5 at 08 UT. 


\section{Appendix B}

\section{Magnetic observatories which appear in Table 1}

List of magnetic observatories of the analysis of the historic events (Table 1).

Table B1. Geographic and magnetic location of magnetic observatories in the analysis of the historic events (Table 1). The fifth column refers to the magnetic latitude according to the IGRF model available closest of the year of the event (when several periods are involved, the magnetic latitude range is given). Column 6 gives information to transform from Universal Time to Magnetic Local Time.

\begin{tabular}{|c|c|c|c|c|c|}
\hline Observatory name & IAGA code & Geographic latitude $\left(^{\circ}\right)$ & Geographic longitude $\left(^{\circ}\right)$ & Magnetic latitude $\left(^{\circ}\right)$ & UT-MLT (h) \\
\hline Apia & API & -13.80 & 188.22 & -16.0 & -11.45 \\
\hline Ashkhabad & $\mathrm{ASH}$ & 37.95 & 58.10 & 30.3 & +3.87 \\
\hline Coimbra & COI & 40.22 & 351.58 & 44.9 & -0.56 \\
\hline Colaba & CLA & 19.06 & 72.35 & 9.9 & +4.82 \\
\hline Cuajimalpa & CUA & 20.53 & 259.47 & 29.9 & -6.70 \\
\hline Fresno & FRN & 37.09 & 240.28 & 43.6 & -7.99 \\
\hline Greenwich & GRW & 51.48 & 0.00 & $54.1 \pm 0.1$ & 0.00 \\
\hline Hartland & HAD & 51.00 & 355.52 & 54.4 & -0.30 \\
\hline Hermanus & HER & -34.43 & 19.23 & $-33.4 \pm 0.1$ & +1.28 \\
\hline Hurbanovo & HRB & 47.86 & 18.19 & 47.1 & +1.21 \\
\hline Kakioka & KAK & 36.23 & 140.18 & $26 \pm 0.3$ & +9.35 \\
\hline San Fernando & SFS & 36.67 & 354.06 & 41 & -0.40 \\
\hline SanJuan & SJG & 18.11 & 293.85 & 29.5 & -4.41 \\
\hline San Pablo-Toledo & SPT & 39.55 & 355.65 & $43.2 \pm 0.2$ & -0.29 \\
\hline Tbilisi & TFS & 42.08 & 44.70 & 36.4 & +2.98 \\
\hline Tihany & THY & 46.90 & 17.89 & 45.8 & +1.19 \\
\hline Tucson & TUC & 32.18 & 249.27 & 40.3 & -7.38 \\
\hline Vieques & VQS & 18.15 & 294.55 & 29.4 & -4.36 \\
\hline
\end{tabular}




\section{Appendix C}

\section{Figures for extreme events in Table 3}

Figures for all extreme events in Table 3 of the manuscript except that of the event \#7, which is included as an example event of data processing in Section 3, and that of the event \#2, included in Section 4. Each figure has the same format as Figure 7.

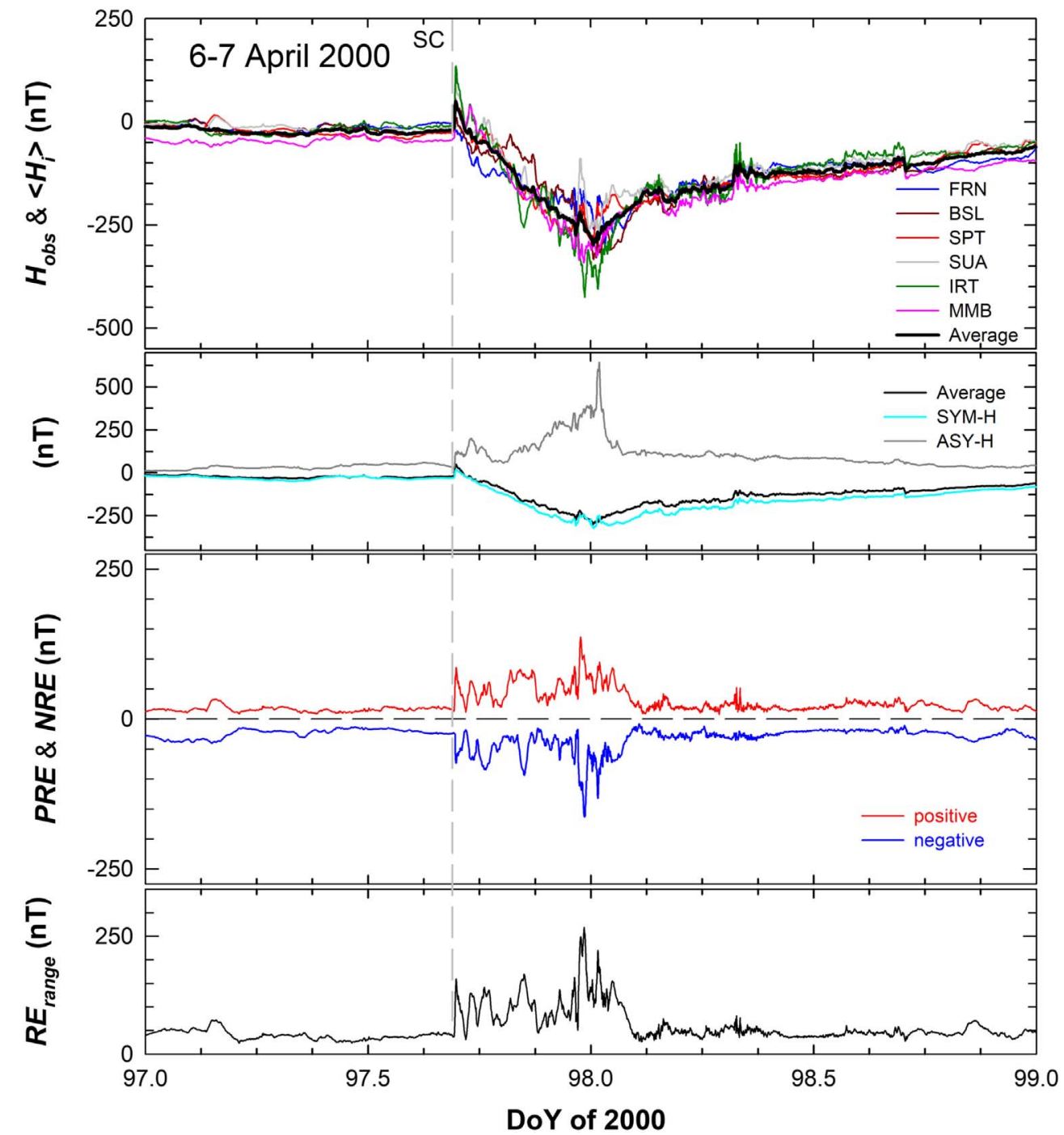

Fig. C.1. The event on 6-7 April 2000 (event \#1). Top panel: Magnetic disturbance, $H_{o b s}$, at the six mid-latitude observatories (in colors) and the average of the disturbance, $\left\langle H_{i}\right\rangle$ (in black); Second panel: $\left\langle H_{i}\right\rangle$ (in black), $S Y M-H$ index (in cyan) and $A S Y-H$ index (in dark gray); Third panel: Positive (red) and Negative (blue) Residual Envelopes; Bottom panel: Residual range obtained as the difference between the Positive and Negative Residual Envelopes. Grey dashed line marks the SSC in the period plotted. 
E. Saiz et al.: J. Space Weather Space Clim. 2021, 11, 35

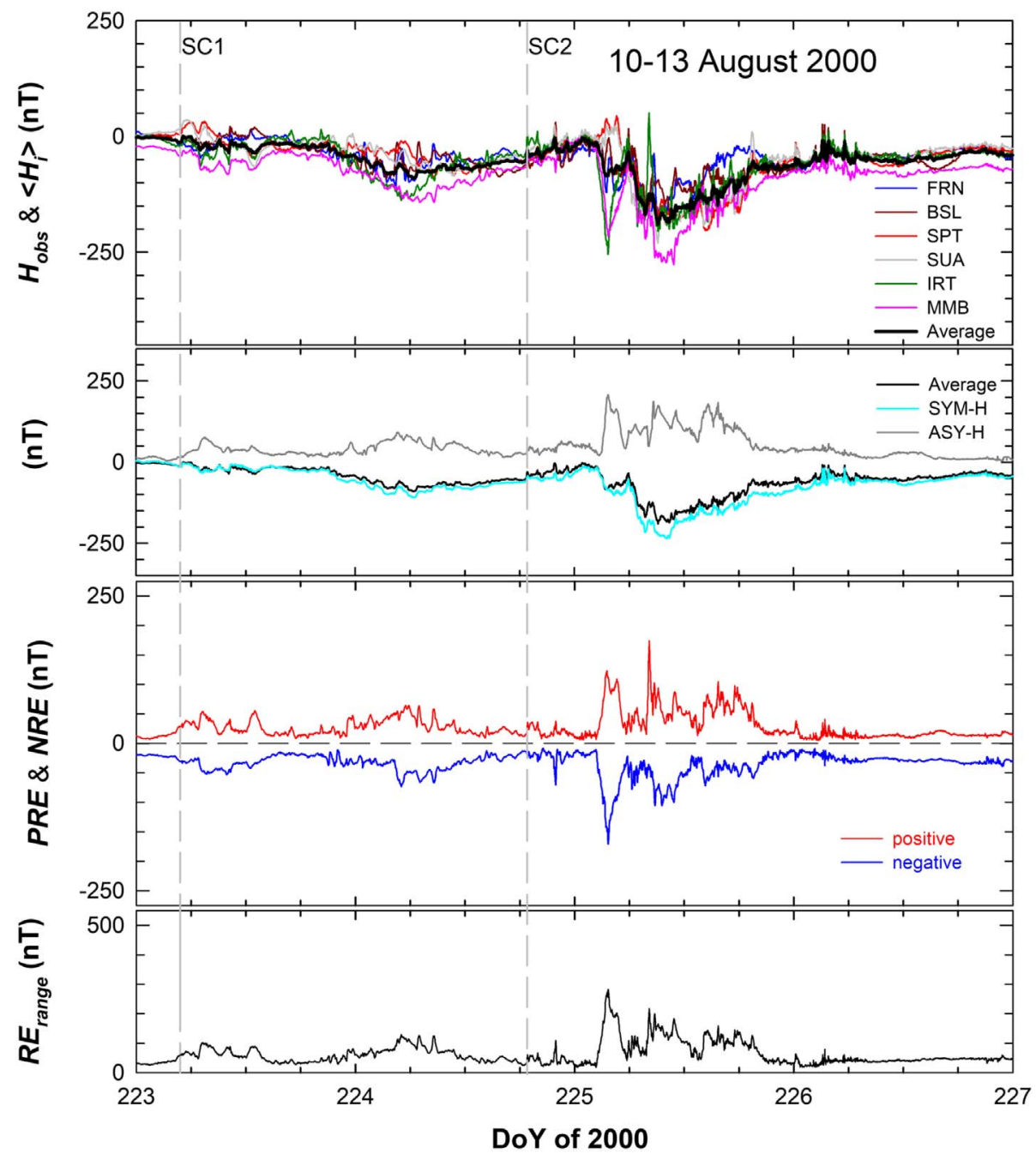

Fig. C.2. The event on 10-13 August 2000 (event \#3). 
E. Saiz et al.: J. Space Weather Space Clim. 2021, 11, 35

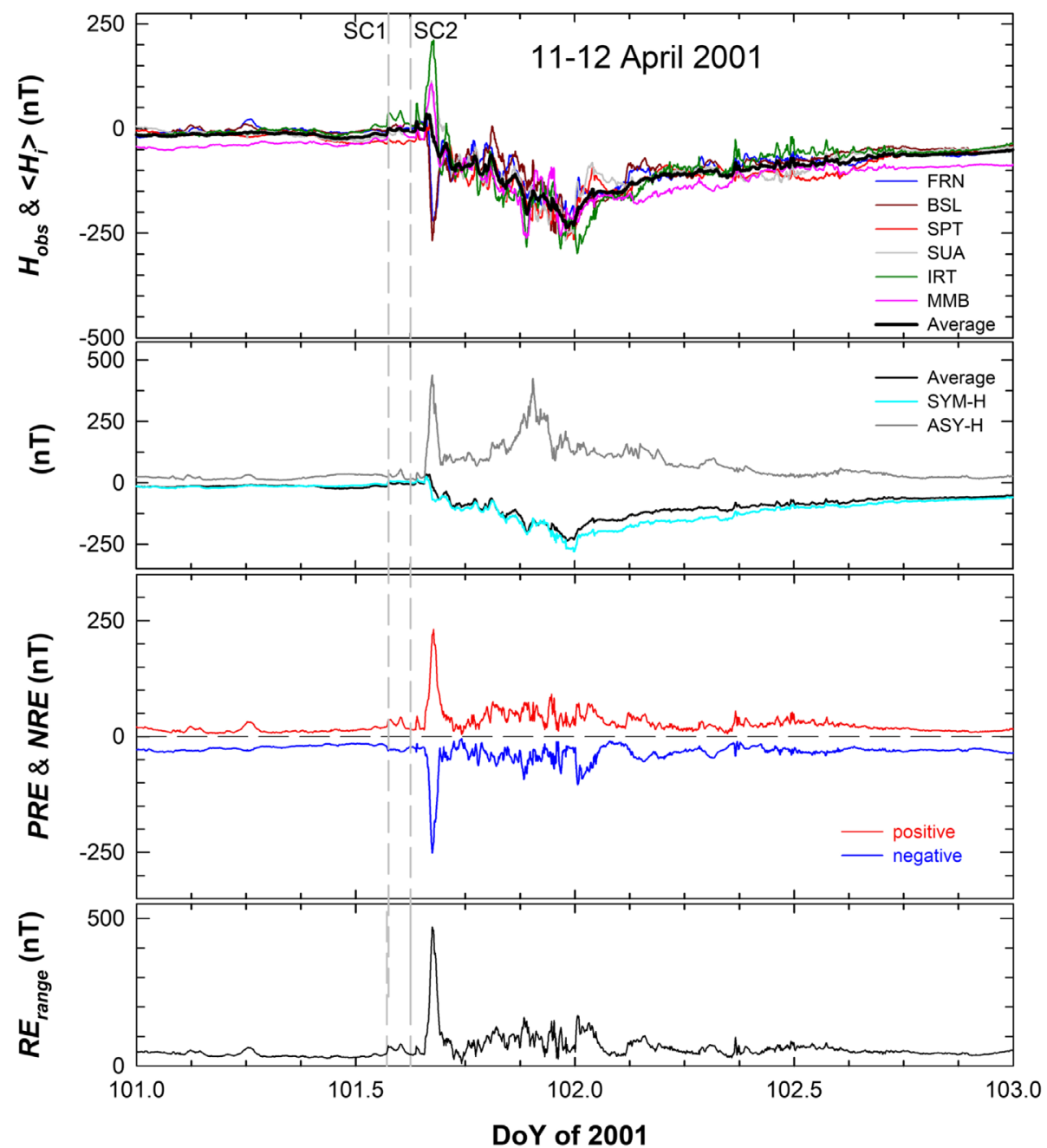

Fig. C.3. The event on 11-12 April 2001 (event \#4). 
E. Saiz et al.: J. Space Weather Space Clim. 2021, 11, 35

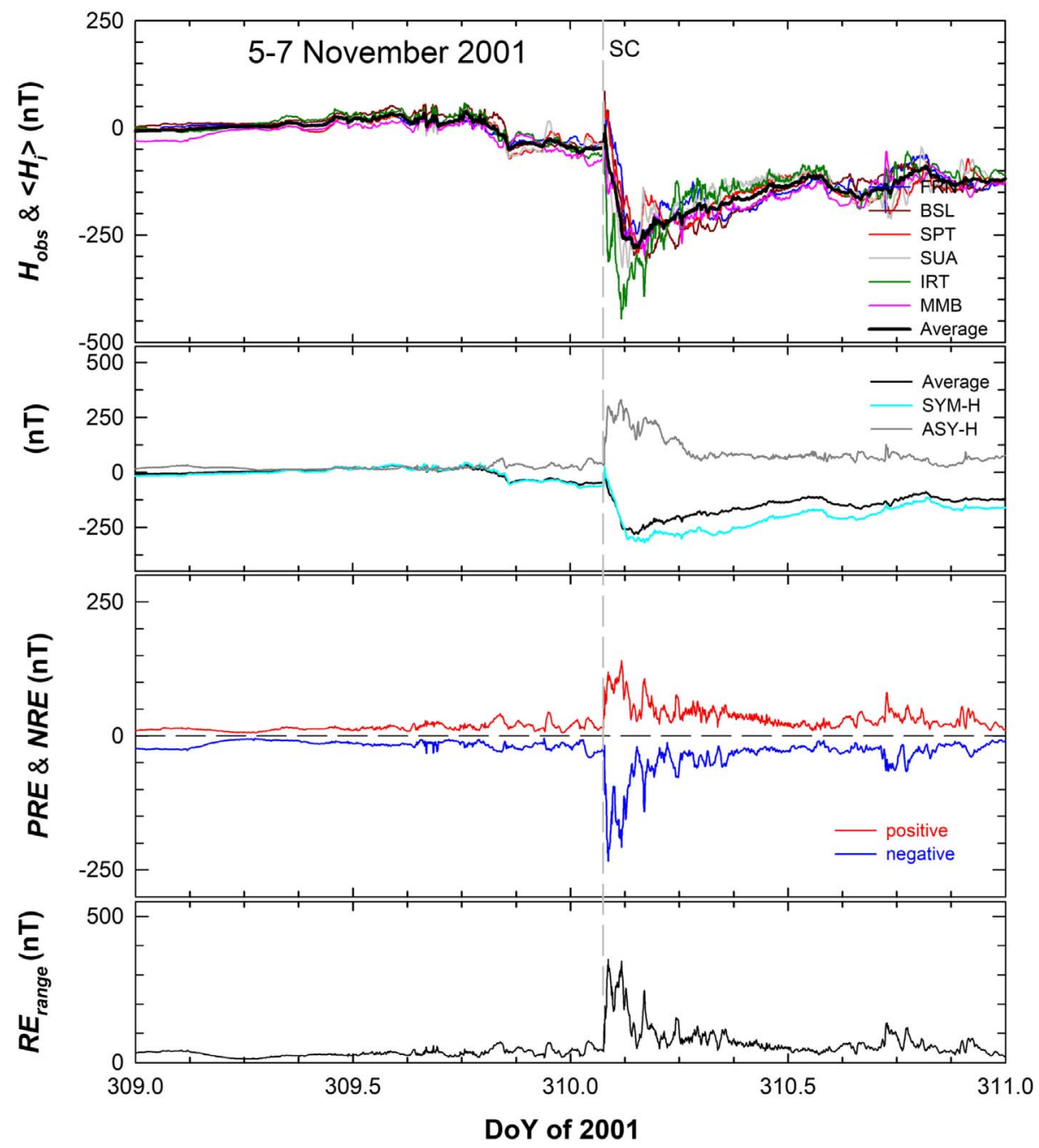

Fig. C.4. The event on 5-7 November 2001 (event \#5). 
E. Saiz et al.: J. Space Weather Space Clim. 2021, 11, 35

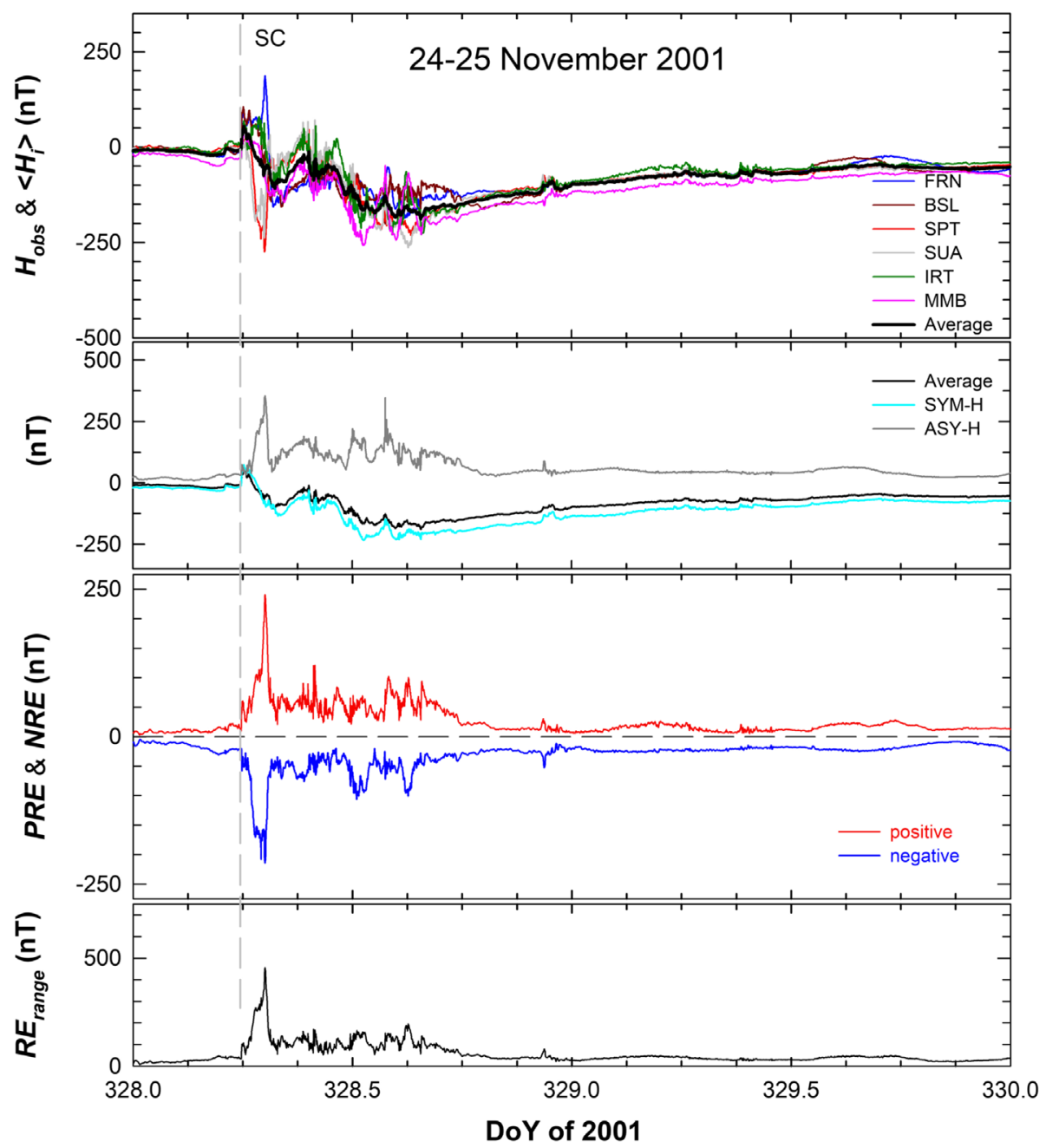

Fig. C.5. The event on 24-25 November 2001 (event \#6). 
E. Saiz et al.: J. Space Weather Space Clim. 2021, 11, 35

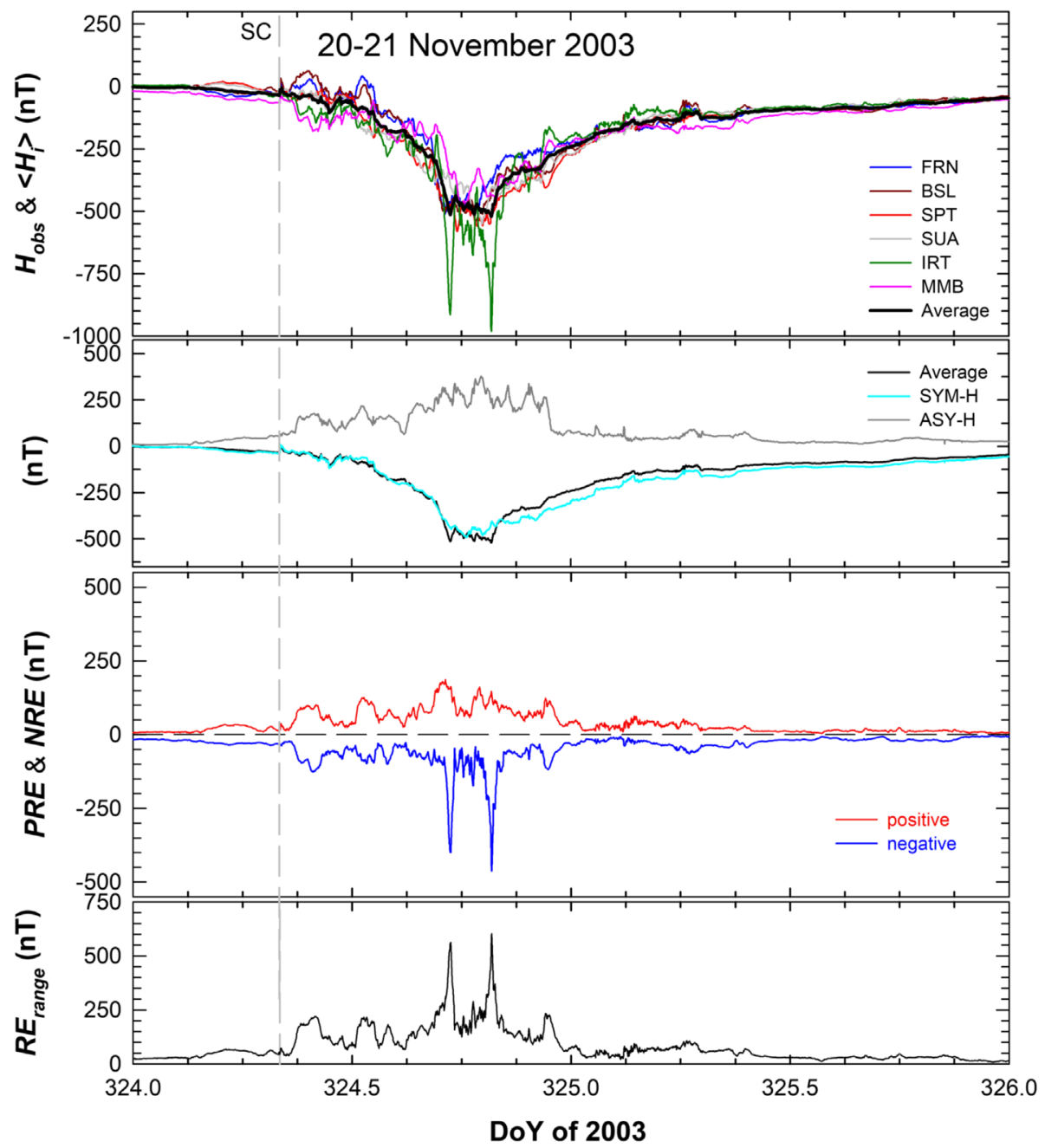

Fig. C.6. The event on 20 November 2003 (event \#8). 
E. Saiz et al.: J. Space Weather Space Clim. 2021, 11, 35

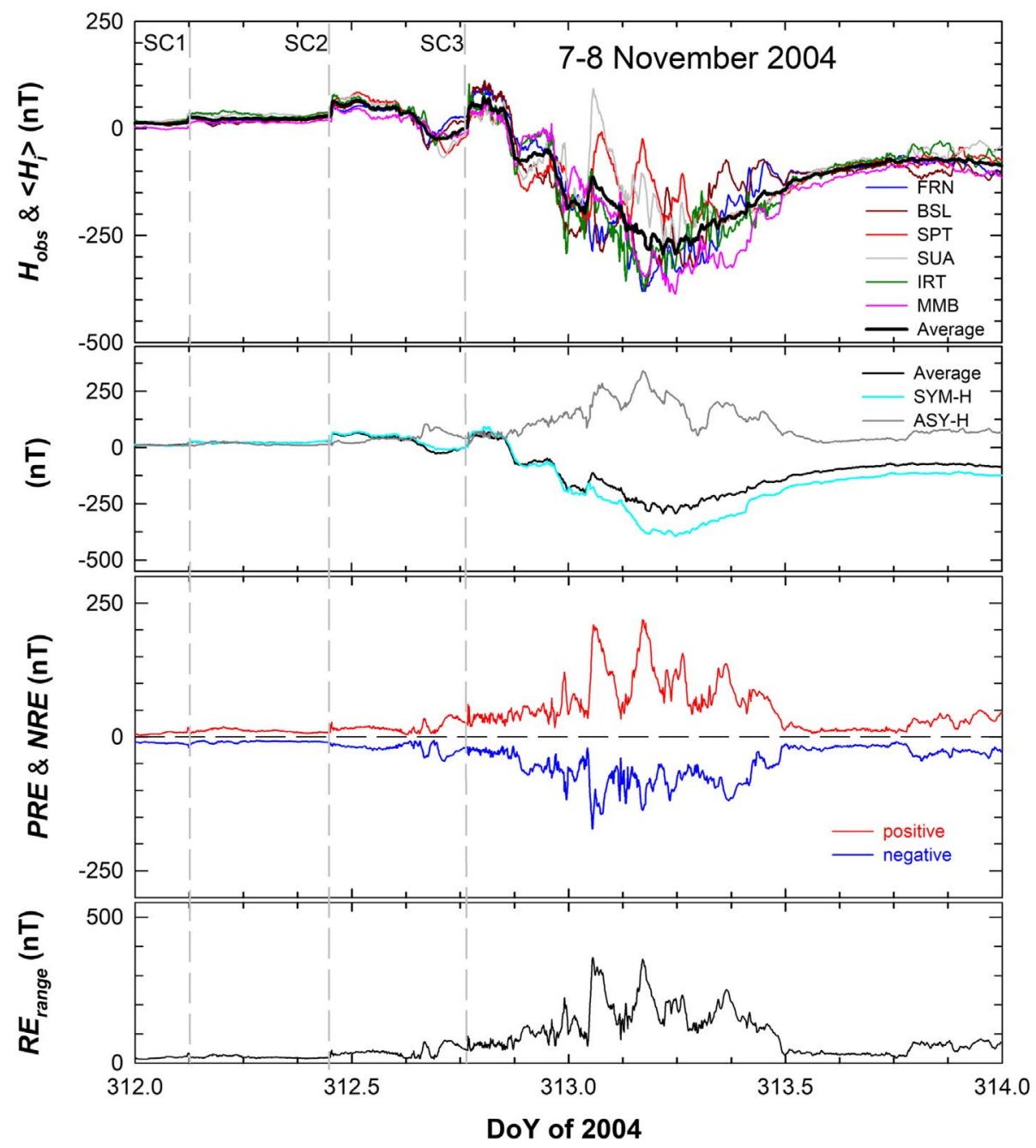

Fig. C.7. The event on 7-8 November 2004 (event \#9). 


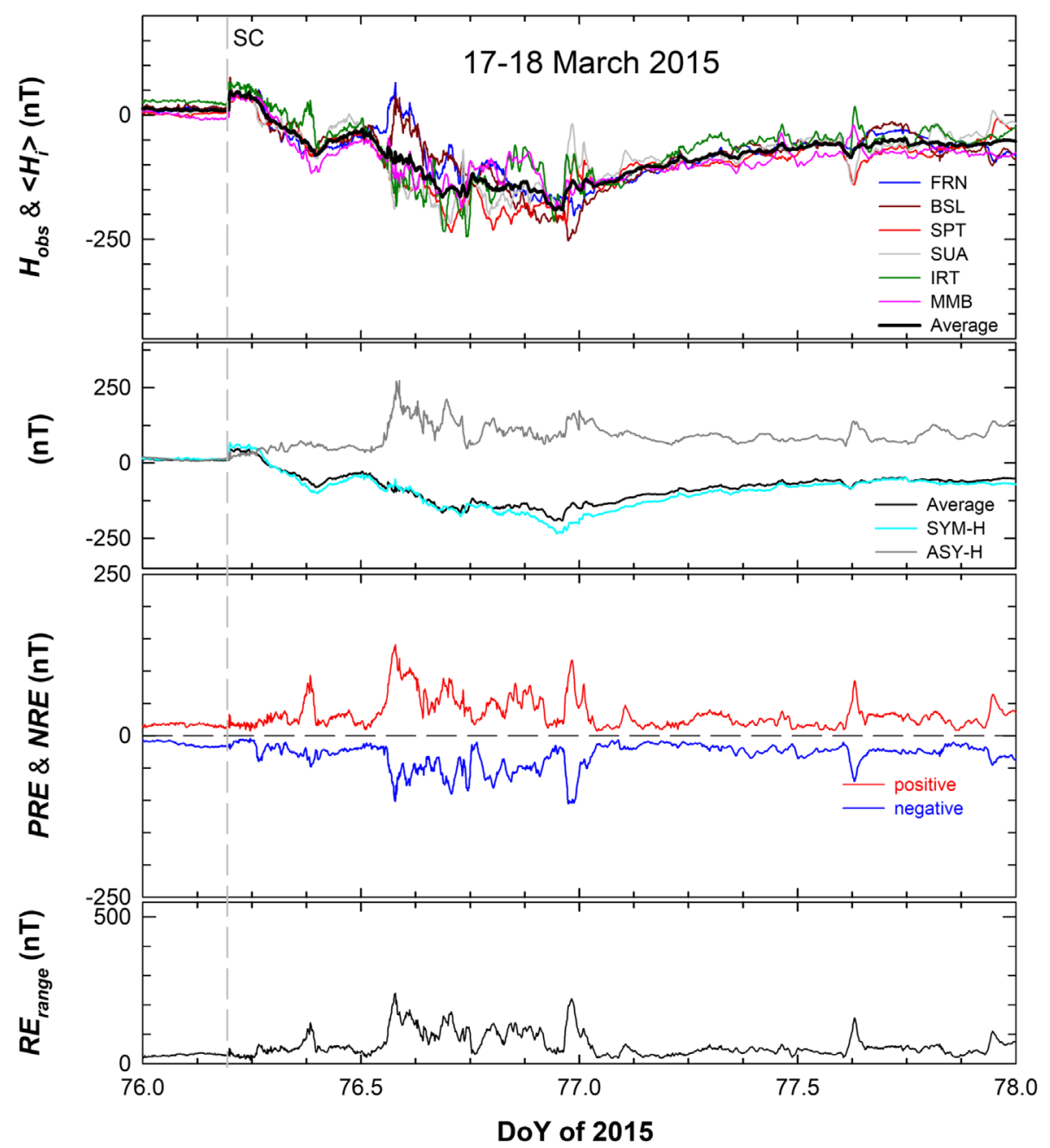

Fig. C.8. The event on 17-18 March 2015 (event \#10).

Cite this article as: Saiz E, Cid C \& Guerrero A 2021. The relevance of local magnetic records when using extreme space weather events as benchmarks. J. Space Weather Space Clim. 11, 35. https://doi.org/10.1051/swsc/2021018. 